\title{
Measurement of exhaled nitric oxide and serum surfactant protein D levels for monitoring radiation pneumonitis following thoracic radiotherapy
}

\author{
HIDEYA YAMAZAKI $^{1}$, NORIHIRO AIBE ${ }^{1}$, SATOAKI NAKAMURA $^{1}$, NAOMI SASAKI $^{1}$, \\ GEN SUZUKI $^{1}$, KEN YOSHIDA $^{2}$, KEI YAMADA ${ }^{1}$, MASAHIKO KOIZUMI ${ }^{3}$, TAICHIRO ARIMOTO ${ }^{4}$, \\ YOSHINOBU IWASAKI $^{4}$, YOSHIKO KANEKO ${ }^{4}$ and KOICHI TAKAYAMA ${ }^{4}$
}

\begin{abstract}
${ }^{1}$ Department of Radiology, Graduate School of Medical Science, Kyoto Prefectural University of Medicine Kyoto, Kyoto 602-8566; ${ }^{2}$ Department of Radiology, Osaka Medical College, Takatsuki, Osaka 569-8686; ${ }^{3}$ Department of Radiation Oncology, Division of Health Sciences, Osaka University, Suita, Osaka 565-0871; ${ }^{4}$ Department of Pulmonary Medicine, Graduate School of Medical Science, Kyoto Prefectural University of Medicine Kyoto, Kyoto 602-8566, Japan
\end{abstract}

Received July 7, 2016; Accepted June 23, 2017

DOI: $10.3892 / 01.2017 .6691$

\begin{abstract}
The present study aimed to examine the role of exhaled nitric oxide (eNO) and serum surfactant protein D (SP-D) level in the determination of radiation pneumonitis (RP) after thoracic radiotherapy (RT). The study included 34 treatments for 33 patients, including 16 three-dimensional conformal and 18 stereotactic body RT treatments. eNO levels were measured prior to RT, immediately subsequent to RT, every week during the RT course and at $1,3,6$, 9 and 12 months following the treatment. The therapy reduced the eNO from $24.3 \pm 12.8 \mathrm{ppb}$ prior to RT to $19.0 \pm 10.4$ ppb immediately subsequent to RT $(\mathrm{P}=0.04)$. A total of 5 patients $(14 \%)$ developed symptomatic RP of grade 2 or higher 3-5 months later, and exhibited an eNO elevation of $2.1 \pm 0.68$-fold the minimum value, whereas the RP- group exhibited $1.4 \pm 0.6$-fold elevation $(\mathrm{P}=0.02)$. The sensitivity of a cut-off of a 1.4-fold increase in the eNO ratio at the onset of RP was $100 \%$; however, the specificity was $52 \%$, and no predictive alterations to eNO levels were observed prior to the onset of RP. RT was associated with an elevated serum SP-D level at 3-6 months after RT. There was a statistically significant difference in the initial serum SP-D level between $\mathrm{RP}^{+}$and $\mathrm{RP}^{-}$patients. In conclusion, obtaining the eNO ratio was a useful RP monitoring tool but did not predict RP occurrence in the present setting, whereas serum SP-D level may be a potential predictor for the detection of RP risk.
\end{abstract}

Correspondence to: Dr Hideya Yamazaki, Department of Radiology, Graduate School of Medical Science, Kyoto Prefectural University of Medicine Kyoto, 465 Kajiicho Kawaramachi Hirokoji, Kamigyo Kyoto, Kyoto 602-8566, Japan

E-mail: hideya10@hotmail.com

Key words: thoracic radiotherapy, radiation pneumonitis, exhaled NO

\section{Introduction}

Nitric oxide is a multifunctional biological mediator and an active participant in proinflammatory and bronchodilatory biological processes (1). The measurement of exhaled nitric oxide (eNO) levels is a simple, non-invasive technique; it is used to monitor airway inflammation in patients with asthma and as an indicator for steroid usage $(2,3)$. eNO assessment is also useful in other diseases, e.g., chronic obstructive pulmonary disease (4), pulmonary hypertension (3) and scleroderma (5). We previously hypothesized that eNO may be a predictor for radiation pneumonitis (RP) following the thoracic radiotherapy (RT) of patients with lung cancer (6). However, when the original study was performed, two-dimensional RT planning and a large eNO assay-machine were utilized. The equipment required a long time for initial calibration, making the task impractical; this ultimately resulted in the discontinuation of the study. In the 21 st century, three-dimensional computed tomography (CT)-guided conformal radiation therapy (3D-CRT) has become a standard treatment. Additionally, advanced RT technology, including stereotactic body RT (SBRT), is now available in clinical settings. Improved, small-size equipment for measuring eNO concentrations has been developed and is also available in clinical practice. These developments made it possible to re-examine eNO levels using the new equipment in order to observe the effects of modern RT techniques. Additionally, there are several biomarkers proposed for RP monitoring, including surfactant protein D (SP-D) $(7,8)$. A simultaneous, prospective examination of the levels of this biomarker was performed. The overall aim of the study was to confirm the results of our previous study and examine the possible extension of the methods for monitoring RP after advanced RT treatments.

\section{Materials and methods}

Patients. Between February 2012 and July 2015, the eNO levels from the 34 thoracic RT treatments of 33 patients 
(25 male, 8 female), with a median age of 78 (range, 37-87), were assessed in the Department of Radiology, Graduate School of Medical Science, Kyoto Prefectural University of Medicine (Kyoto, Japan). Patients were staged according to the Union for International Cancer Control, 7th edition (9). A total of 16 patients received 3D-CRT (including patients at primary lung cancer stage T1N0, 2; T1N1, 1; T1N2, 1; T2N0, 1; T3N0, 1; T3N1 1; T3N3, 1; T4N2, 1; T4N3, 1; rN1 2 and rN3 1, in addition to 1 with $\mathrm{rN} 1$ esophageal cancer and 2 with metastatic lung cancer) and 18 received SBRT (including 13 with primary lung cancer, including T1N0, 8; T2N0, 5; 6 with metastatic lung cancer). One patient underwent thoracic RT twice, including initial 3D-CRT for primary esophageal cancer and SBRT for metastatic lung cancer. Patients were ineligible if they had an Eastern Cooperative Oncology Group performance status $>2$ (10), active systemic or pulmonary infection, asthma or synchronous malignancy within 2 years of enrollment.

$3 D$-CRT procedure. For 3D-CRT, the patients were placed in a supine position with arms above the head. Target volumes were delineated on a planning CT scan with $2.5 \mathrm{~mm}$ slices. The gross tumor volume (GTV), clinical target volume (CTV), and planning target volume (PTV) were obtained according to the definitions of the International Commission on Radiation Units and Measurements (11). The GTV included tumor and lymph node tissue visualized using a CT and positron emission tomography (PET)-CT scan. PTV, including set-up margin and internal target volume, was obtained using a $2-10 \mathrm{~mm}$ 3D-expansion from CTV. The median prescribed dose was $60 \mathrm{~Gy}$ in 30 fractions. Concurrent chemotherapy (cisplatin with pemetrexed, 5 patients; carboplatin with paclitaxel, 2 patients; cisplatin with fluorouracil, 1 patient) was administered only for patients receiving 3D-CRT.

SBRT procedure. SBRT was performed using an immobilization device Body FIX ${ }^{\circledR}$ Vacuum Pump P2 system (Medical Intelligence Medizintechnik GmbH, Schwabmünchen, Germany), with the patient in a supine position. All patients underwent 3 simulation CT scans (Aquilion 64; Toshiba Medical Systems Ltd., Tokyo, Japan) in three phases, i.e., free breathing, inhalation and exhalation during shallow breathing. The GTV in each phase was delineated using lung CT (window level, $550 \mathrm{HU}$; width, 1,600 HU) and a radiation treatment contouring system (XiO; Elekta AB, Stockholm, Sweden). GTVs of the 3 phases were fused and expanded as a PTV with a margin of $5 \mathrm{~mm}$ in the anteroposterior and right-left directions and $5-10 \mathrm{~mm}$ in the superior-inferior and anterior-posterior directions (typically $5 \mathrm{~mm}$ ). The following structures were contoured as organs at risk: Spinal cord, esophagus, lung and others as required by the situation (e.g., the heart, ipsilateral bronchus, liver and bowels). The treatment was generally delivered so that $>95 \%$ of the PTV received the prescribed dose of $50 \mathrm{~Gy}$. The median prescribed dose was $48 \mathrm{~Gy}$ in 4 fractions.

Irradiation was applied using 4-, 6-, and 10-mV photon beams from accelerators (Elekta Synergy; Elekta AB) with multileaf linear collimators. Lung toxicity grade 2 (symptomatic RP with image abnormality) or higher, according to Common Terminology Criteria of Adverse Events v4.0 (12), was regarded as RP; one patient exhibited moderate fibrotic lung but did not suffer symptomatic radiation pneumonitis.
The toxicity assessment was performed by physicians from several departments, including a diagnostic radiologist, pulmonologist and radiation oncologist. All patients had signed an informed consent before registration in the observational study, which was approved by the Institutional Review Board of Kyoto Prefectural University of Medicine (approval no. PBMR-C-988-1).

eNO measurements. eNO levels were measured using a chemiluminescence analyzer (Aerocrine NIOX; Circassia AB, Uppsala, Sweden). The results were expressed as the mean of triplicate measurements. eNO measurements were performed prior to RT, immediately subsequent to RT, every week during RT, and 1, 3, 6, 9 and 12 months later. Additional measurements were conducted if RP was suspected. The eNO ratio was calculated as current eNO value/minimum eNO value during RT. Serum SP-D level was also examined prior to RT and 1, 3,6 , and 12 months after RT as described previously $(7,8)$. To obtain control eNO values, the eNO levels of 17 healthy volunteers were assessed.

Statistical analysis. All statistical analyses were performed using StatView 5.0 statistical software (SAS Institute, Inc., Cary, NC, USA). Percentages were compared using the $\chi^{2}$ test; comparisons between time points within the same group were compared using Student's paired t-test and the Mann-Whitney $\mathrm{U}$ test was used for other comparisons. $\mathrm{P}<0.05$ was considered to indicate a statistically significant difference.

\section{Results}

$e N O$. The mean \pm standard deviation eNO level prior to RT was $24.3 \pm 12.8 \mathrm{ppb}$; it decreased to $19.0 \pm 10.4 \mathrm{ppb}$ following treatment (immediately subsequent to RT, $\mathrm{P}=0.04$; Fig. $1 \mathrm{~A}$ ). In the 3D-CRT group, a statistically significant difference between the initial value and the value at 4 weeks during RT was identified (Fig. 1B). Patients with a primary lung tumor $(n=25)$ exhibited an initial eNO level $(23.4 \pm 13.0 \mathrm{ppb})$ higher than healthy volunteers $(15.7 \pm 8.4 \mathrm{ppb})$ to an extent that bordered on significance $(\mathrm{P}=0.06)$. As the RT treatment reduced eNO levels, the eNO ratio was used instead of the crude eNO value to examine the variation thereafter.

A total of 5 patients (14\%) exhibited RP of grade 2 or higher, including 1 patient receiving SBRT, which developed 3-5 months after RT (median 3 months). A comparison of basic patient characteristics for $\mathrm{RP}^{+}$and $\mathrm{RP}^{-}$patients is presented in Table I. In patients receiving 3D-CRT, the $\mathrm{RP}^{+}$group exhibited a higher lung irradiation dose (including mean lung dose and V20 values) than the $\mathrm{RP}^{-}$group $(\mathrm{P}<0.01$; Table I). There was no difference between eNO levels in the $\mathrm{RP}^{+}$group $(19.7 \pm 6.68 \mathrm{ppb}$ prior to RT, $15.93 \pm 8.98 \mathrm{ppb}$ immediately subsequent to RT) and the RP $\mathrm{P}^{-}$group $(24.4 \pm 13.7 \mathrm{ppb}$ prior to RT, $19.6 \pm 10.7 \mathrm{ppb}$ immediately subsequent to RT) during the examined periods. The eNO ratio increased at the onset of symptomatic RP; the symptomatic $\mathrm{RP}^{+}$group exhibited a $2.1 \pm 0.68$-fold elevation in the eNO ratio at 3-5 months, compared with a 1.4 \pm 0.6 -fold elevation in the $\mathrm{RP}^{-}$group $(\mathrm{P}=0.02$; Fig. $1 \mathrm{C})$. However, in $\mathrm{RP}^{+}$ patients, there was no predictive elevation in the crude eNO values or eNO ratio prior to the onset of RP. Accordingly, an eNO ratio of 1.4 was used as the threshold ratio for RP; this 


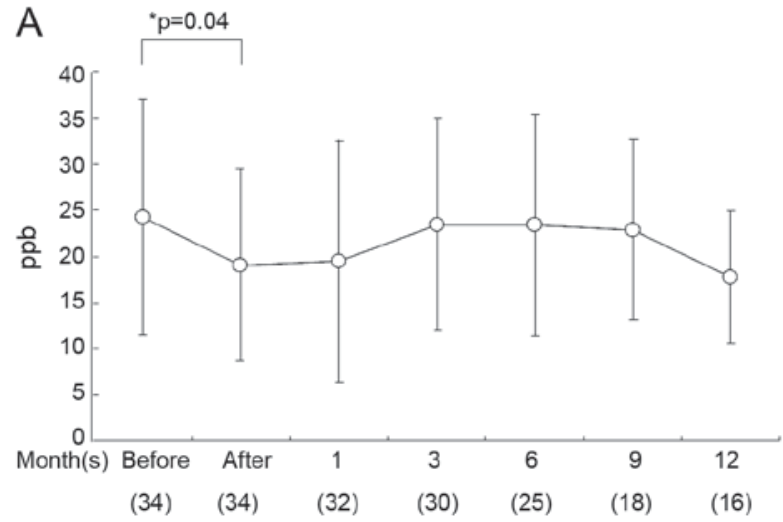

(No. of case examined)

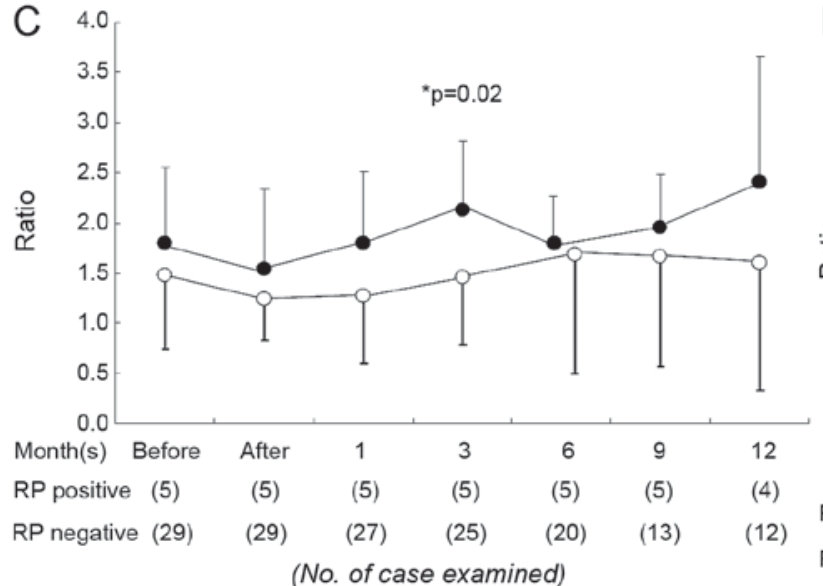

(No. of case examined)

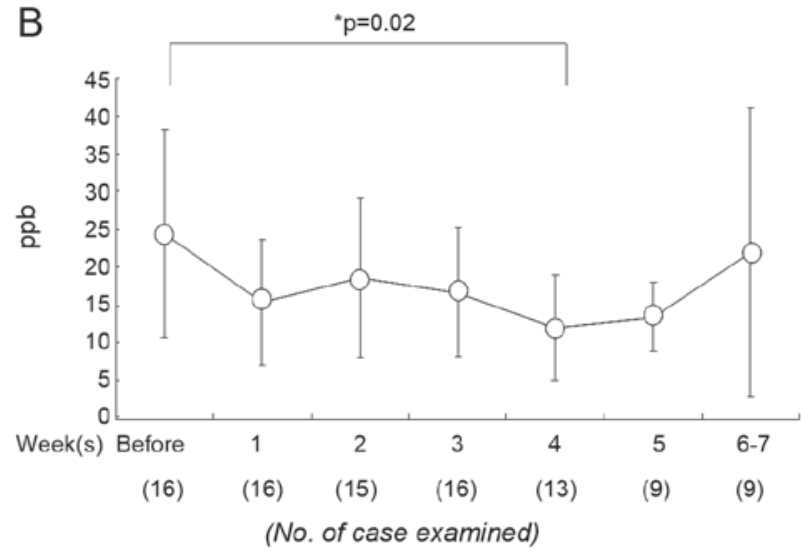

D

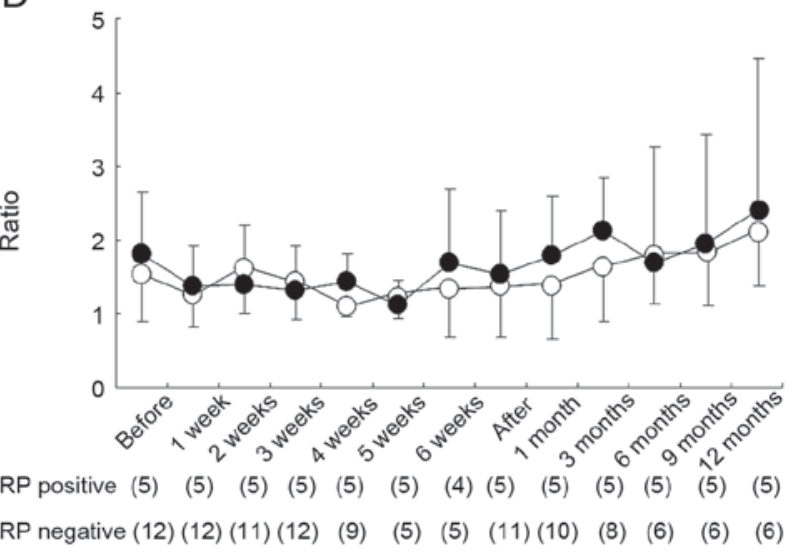

(No. of case examined)

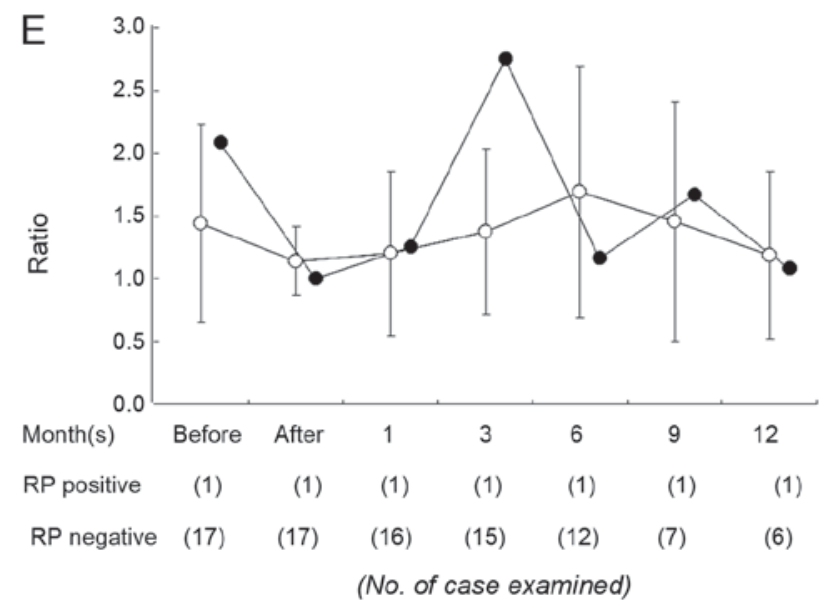

Figure 1. Level of eNO prior to, during and subsequent to RT. (A) Gross eNO levels of all patients. Immediately subsequent to RT, eNO was reduced. (B) Gross eNO levels in the 3D-CRT subgroup. eNO was reduced at week 4 of RT. (C) eNO ratio stratified by RP status. eNO ratio was calculated as eNO/minimum eNO value during RT. (D) eNO ratio stratified by RP status in the 3D-CRT subgroup. (E) eNO ratio stratified by RP status in the stereotactic body RT subgroup. The black circles represent the $\mathrm{RP}^{+}$group and white circles represent the $\mathrm{RP}^{-}$group, in the stratified graphs. eNO, exhaled nitric oxide; RT, radiotherapy; 3D-CRT, three-dimensional conformal radiation therapy; RP, radiation pneumonitis.

was associated with $100 \%$ sensitivity and $52 \%$ specificity for indicating the occurrence of RP.

Serum SP-D. Serum SP-D level was also examined to assess its association with RP. The RT treatment did not significantly alter serum SP-D levels $(67.8 \pm 49.4 \mathrm{ng} / \mathrm{ml}$ prior to RT, $67.5 \pm 37.8 \mathrm{ng} / \mathrm{ml}$ immediately subsequent to RT; Fig. 2A). However, the SP-D level was elevated at 3-6 months after RT (Fig. 2A). The $\mathrm{RP}^{+}$group exhibited a significantly higher serum SP-D level than the RP- group prior to RT $\left(\mathrm{RP}^{+}, 105 \pm 46 \mathrm{ng} / \mathrm{ml}\right.$,
$\mathrm{RP}^{-}, 61 \pm 47 \mathrm{ng} / \mathrm{ml} ; \mathrm{P}=0.03$; Fig. 2B). At 6 months later, the levels of serum SP-D remained significantly different between these groups ( $\mathrm{P}=0.02$; Fig. 2B). Serum SP-D level prior to RT exhibited $83 \%$ specificity and $40 \%$ sensitivity for RP prediction (Table II). None of the patients with a serum SP-D level of $\leq 50 \mathrm{ng} / \mathrm{ml}$ prior to RT experienced RP (Fig. 3). The criteria of an eNO ratio $<1.4$ and SP-D $>109 \mathrm{ng} / \mathrm{ml}$ provided $100 \%$ sensitivity and $48 \%$ specificity for the prediction of RP, whereas the criteria of an eNO ratio $<1.4$ or SP-D $>109 \mathrm{ng} / \mathrm{ml}$ provided $40 \%$ sensitivity and $100 \%$ specificity. 
Table I. Characteristics and treatments of the patients.

\begin{tabular}{|c|c|c|c|c|}
\hline \multirow[b]{2}{*}{ Characteristic } & \multicolumn{3}{|c|}{ Radiation pneumonitis status } & \multirow[b]{2}{*}{ P-value } \\
\hline & All patients & Negative & Positive & \\
\hline Total patients, $\mathrm{n}$ & $33^{\mathrm{a}}$ & $28^{\mathrm{a}}$ & 5 & \\
\hline Age, median (range) years & $78(37-87)$ & $76(62-80)$ & $78(37-87)$ & 0.63 \\
\hline Sex, n (\%) & & & & 0.41 \\
\hline Female & $8(24)$ & $8(29)$ & $0(0)$ & \\
\hline Male & $25(76)$ & $20(71)$ & $5(100)$ & \\
\hline Disease, n (\%) & & & & 0.85 \\
\hline Primary lung cancer & $25(76)$ & $22(79)$ & $1(20)$ & \\
\hline Adenocarcinoma & $17(51)$ & & & \\
\hline Squamous cell carcinoma & $2(6)$ & & & \\
\hline Others & $6(18)$ & & & \\
\hline Esophageal cancer & $2(6)$ & $2(7)$ & $0(0)$ & \\
\hline Metastatic lung cancer & $5(15)$ & $5(18)$ & $0(0)$ & \\
\hline \multicolumn{5}{|l|}{ Treatment, n (\%) } \\
\hline SBRT & $18(53)$ & $17(59)$ & $1(20)$ & 0.26 \\
\hline 48 Gy in 4 fractions & $15(44)$ & & & \\
\hline 50 Gy in 5 fractions & $2(6)$ & & & \\
\hline 60 Gy in 10 fractions & $1(3)$ & & & \\
\hline 3D-CRT & $16(47)$ & $12(41)$ & $4(80)$ & \\
\hline 60 Gy in 20 fractions & $2(6)$ & & & \\
\hline 69 Gy in 23 fractions & $2(6)$ & & & \\
\hline 66 Gy in 12 fractions & $1(3)$ & & & \\
\hline 50 Gy in 25 fractions & $1(3)$ & & & \\
\hline 54 Gy in 27 fractions & $1(3)$ & & & \\
\hline 60 Gy in 30 fractions & $4(12)$ & & & \\
\hline 65 Gy in 30 fractions & $1(3)$ & & & \\
\hline $67.2 \mathrm{~Gy}$ in 34 fractions & $1(3)$ & & & \\
\hline $64 \mathrm{~Gy}$ in 40 fractions & $2(6)$ & & & \\
\hline 70 Gy in 42 fractions & $1(3)$ & & & \\
\hline Concurrent chemotherapy, $\mathrm{n}(\%)$ & & & & 0.71 \\
\hline Yes & & $6(21)$ & $2(40)$ & \\
\hline No & & $23(79)$ & $3(60)$ & \\
\hline Exhaled NO, median (range) ppb & & $19(13-27)$ & $22(8-60)$ & 0.77 \\
\hline Krebs von den Lungen- 6 , median (range) $\mathrm{U} / \mathrm{ml}$ & & $298(173-567)$ & $372(154-2,066)$ & 0.84 \\
\hline Surfactant protein-D, median (range) $\mathrm{ng} / \mathrm{ml}$ & & $47.6(17.2-207)$ & $95.8(55.1-167)$ & 0.03 \\
\hline \multicolumn{5}{|l|}{ Mean lung dose, median (range) Gy } \\
\hline 3D-CRT & & $8.55(1.84-13.38)$ & $14.49(9.92-16.05)$ & 0.01 \\
\hline SBRT & & $3.08(1.42-5.81)$ & 3.58 & 0.77 \\
\hline \multicolumn{5}{|l|}{$\mathrm{V} 20_{\text {lung }}$, median (range) Gy } \\
\hline 3D-CRT & & $14.49(9.92-16.05)$ & $28.3(17.6-31.8)$ & 0.01 \\
\hline SBRT & & $3.21(1.24-10.09)$ & 6.01 & 0.20 \\
\hline
\end{tabular}

${ }^{a}$ One patient underwent treatment twice, including 3D-CRT and SBRT. NS, not significant; SBRT, stereotactic body radiotherapy; 3D-CRT, three-dimensional conformal radiation therapy.

Among the 5 RP cases, 2 of the patients exhibited a unique pattern of serum SP-D expression. The patients exhibited high serum SP-D level prior to RT; those levels were initially reduced by RT and surged to abnormally high values at the 

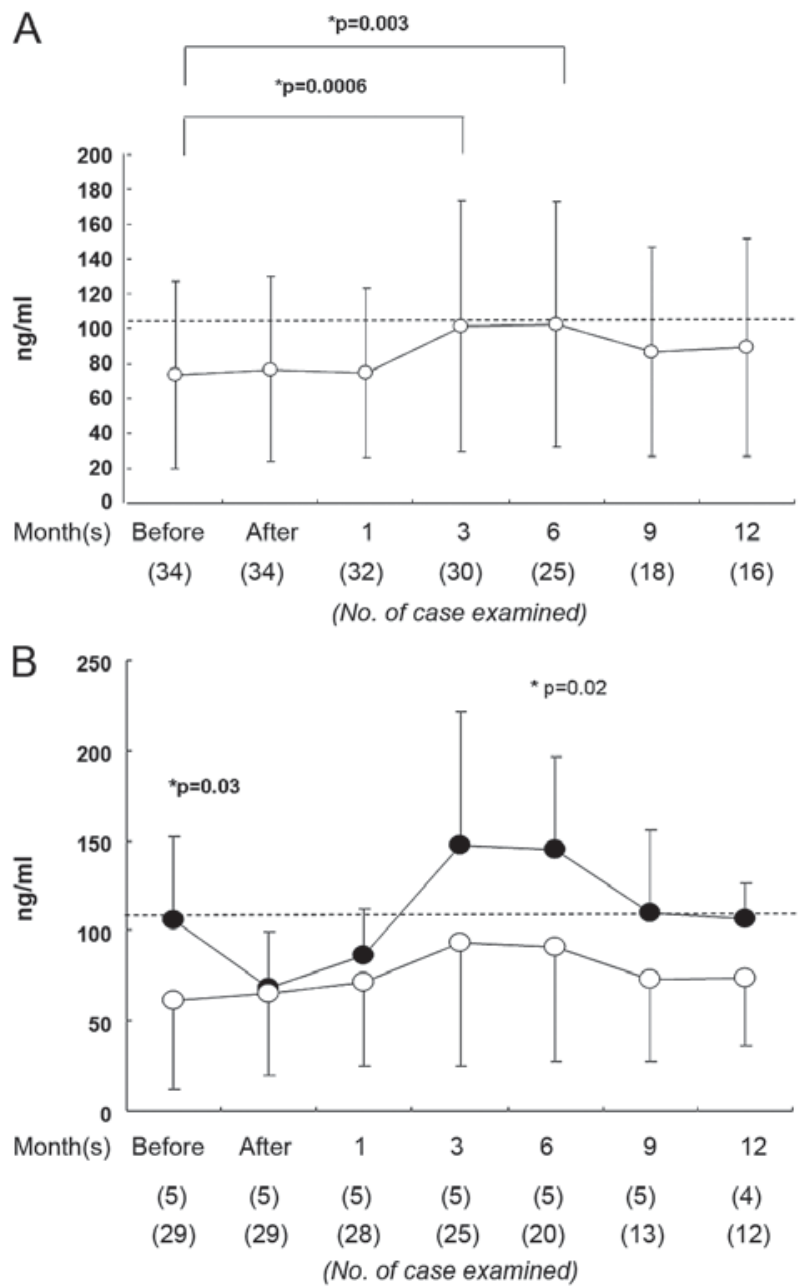

Figure 2. Serum SP-D level prior to, during and subsequent to radiotherapy. (A) Serum SP-D level of all patients. The dashed line depicts the threshold value, $109 \mathrm{ng} / \mathrm{ml}$. (B) Serum SP-D level stratified by RP status. The black circles represent the $\mathrm{RP}^{+}$group and white circles represent the $\mathrm{RP}^{-}$group. SP-D, surfactant protein D; RP, radiation pneumonitis.

onset of RP at 3 months (Fig. 3B). Of the $3 \mathrm{RP}^{+}$patients with low initial SP-D levels, 2 exhibited an increase in serum SP-D to an abnormal level (>109 $\mathrm{ng} / \mathrm{ml})$ a month subsequent to RT. The RT+ patient receiving SBRT (presenting with large cell lung cancer, cT1N0M0) exhibited a gradual elevation in serum SPD and eNO levels with patchy consolidation in CT images immediately prior to RP onset. Following the remission of RP, eNO level decreased to $14 \mathrm{ppb}$ at 6 months.

\section{Discussion}

eNO levels increase in patients with Hodgkin lymphoma and normalize following remission $(13,14)$. A similar effect has been reported for patients with lung cancer, which exhibit increased eNO levels, including in our previous study $(6,15)$. It is established that tumors and surrounding tissues produce NO. In the present study, eNO levels in the patients with primary lung cancer was higher than in normal volunteers to an extent which bordered on significance. This may be explained by the characteristics of the patients in the present study, as they were predominantly patients with early stage lung cancer. Our previous study was concerning predominantly patients with advanced cancer (6).
Serial measurements have demonstrated that eNO diminishes subsequent to chemotherapy (16) and RT (6). A previous study demonstrated that at 8 days subsequent to carboplatin or cisplatin-based chemotherapy, the eNO level decreased by $3.8 \mathrm{ppb}$ in patients with lung cancer and returned to the baseline value after 15 days (16). We previously reported that subsequent to RT or combined chemo-RT in 29 patients, the eNO level had decreased by $35 \%$ immediately subsequent to the completion of 40 Gy RT (6). The decrease in the eNO level observed following the treatment of chest malignancies is caused by cell death in the tumor and the surrounding tissues; this may also be accompanied by a reduction in tumor-associated inflammation, including cytokine production (17). The present study confirmed our previous data, demonstrating that RT reduced the levels of eNO.

RP remains a dose-limiting side effect of RT, impairing the quality of life of patients and potentially leading to mortality (18). The level of the inducible NO synthase (iNOS) expression in alveolar macrophages is associated with the level of eNO (11). Immunohistochemical studies indicate that alveolar macrophages are the major cellular site for NO production $(15,16)$. In mice, the alveolar macrophages produce NO, and the expression of iNOS in alveolar macrophages and epithelial cells is increased, subsequent to irradiation (15). Our previous study demonstrated that irradiation itself does not increase the iNOS mRNA levels in vitro; however, it enhances the expression of iNOS in the presence of cytokines [tumor necrosis factor $\alpha$ and interleukin (IL)-6] (16,19). Additionally, the progression of $\mathrm{RP}$ can be reduced by treatment with an iNOS inhibitor $(16,20)$.

A number of studies have demonstrated the value of eNO measurements for predicting RP. In our previous study, a three-fold increase in the eNO levels during RT treatment was reported in 5 patients. A total of 3/5 of these patients developed RP requiring steroid medication (6). McCurdy et al (20) and Guerrero et al (21) examined 28 patients with esophageal cancer and identified that a 1.5-fold increase in eNO exhibited $100 \%$ sensitivity and specificity for the prediction of RP $(18,20)$. In another study by the same group, the ratio of eNO (the level at the end of RT/pre-RT level) was calculated for 50 lung or esophageal cancer patients. A threshold of 1.4 could perfectly distinguish symptomatic and asymptomatic patients (21). In contrast, Enache et al (17) identified that although the specificity of a $10 \mathrm{ppb}$ increase in eNO level was relatively specific (specificity, 83\%) for predicting RP symptoms in lung cancer patients, the sensitivity was low (18\%). The data of the present study supports their conclusion that 10-ppb elevation from the initial eNO level does not always indicate RP. Their results indicated that the basal level of eNO (i.e., prior to RT) was not a good basis for RP prediction, and that the crude eNO value was not suitable for such estimates (18). Moré et al (22) reported that acute changes in eNO level, defined as percent changes between each pre-fraction and post-fraction measurement, were significantly smaller in $\mathrm{RP}^{+}$than in $\mathrm{RP}^{-}$patients. The observations from the present study confirm that eNO variation may be meaningful in the prediction of RP occurrence. The minimum value during treatment was used as the control level, rather than the initial value. The minimum value may be the result of a reduction in tumor-associated inflammation. In our previous study, RP occurred earlier during RT ( $<2$ months) with a larger extent of eNO level variation; $>3$-fold elevation in comparison with the initial level was identified (6). 
Table II. Stratification based on serum SP-D level prior to RT.

$\mathrm{RP}^{+}$

Serum SP-D level prior to RT

High $(>109 \mathrm{ng} / \mathrm{ml})$

patients, $n$

$6 \quad 2$

of this type
All

\section{n}

6

28

$\mathrm{RP}$

Low $(\leq 109 \mathrm{ng} / \mathrm{ml})$

2 Transient decrease with

resurgence at 1 month

Observation of all cases

Observation of all cases

of this type

$\mathrm{n}$

this type

4

4

Gradual increase

25

High level throughout the examination period

N/A
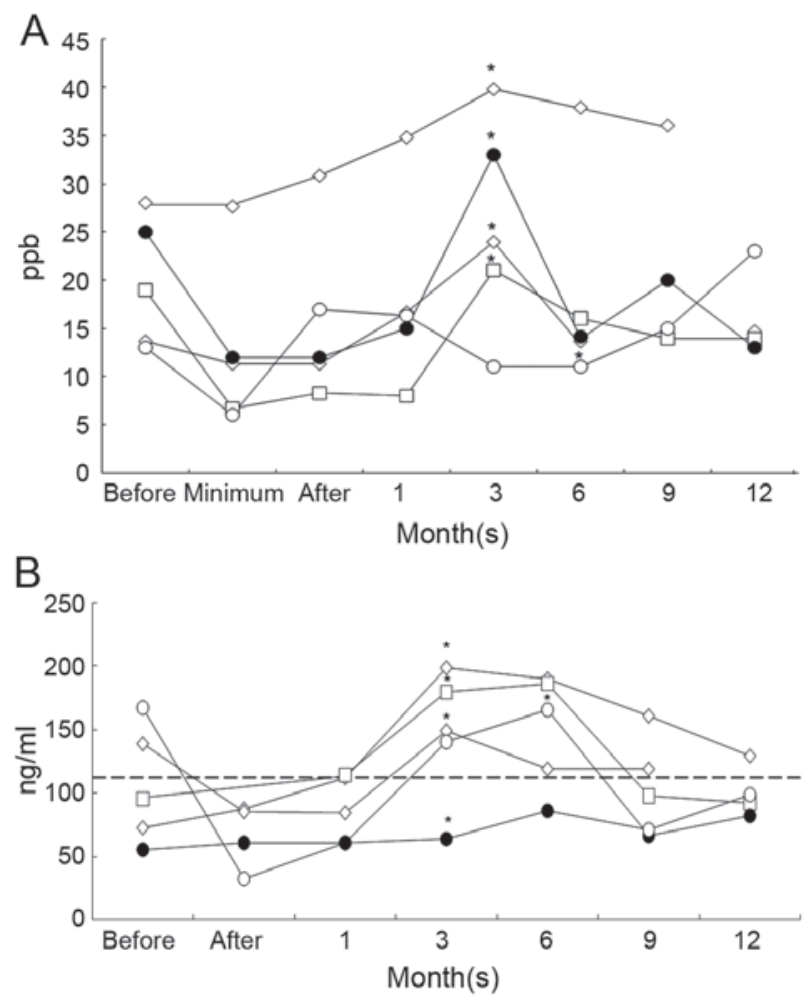

Figure 3. Individual biomarker data for $\mathrm{RP}^{+}$patients. (A) Crude eNO values for the $\mathrm{RP}^{+}$patients. (B) Serum SP-D level. Note the abnormally high levels of serum SP-D level before RT in two patients, that was transiently decreased by RT, and resurgent subsequent to RT. The dashed line depicts the threshold value, $109 \mathrm{ng} / \mathrm{ml}$. Asterisks indicate the time point of RP occurrence. The black circle indicates the patient that underwent stereotactic body RT; note that this patient experienced an increase in eNO, but not in SP-D. RP, radiation pneumonitis; eNO, exhaled nitric oxide; SP-D, surfactant protein D; RT, radiotherapy.

However, in the present study, RP was observed at 3-5 months with a smaller, 1.4-fold increase from the minimum level.

The eNO level could not be used for the prediction of RP in the present study. In the 20th century, RT using 2D planning caused large volumes of tissue to be irradiated and resulted in a RP rate of $\sim 30 \%$. This rate has been reduced to $\sim 10 \%$ with 3D-CRT and SBRT, which irradiate a reduced volume (23). It remains difficult to predict RP by monitoring only during $\mathrm{RT}$ or the following month. The monitoring period for these biomarkers should be extended to $\geq 2$ months after RT treatment. Data from 'before', at 4-5 weeks during RT or 'after' may all be candidates for the minimum value, as in the present study, minimum values occurred at those time points. The most common timing for minimum eNO as determined from the present study was 4 weeks according to Fig. 1B; calculating eNO ratio by measuring at that time point may be useful.

There are a number of reports that serum SP-D is a useful biomarker for RP prediction $(7,8,24-26)$. Yamashita et al $(8,19)$ have reported that patients presenting with an interstitial pneumonitis shadow in CT imaging and a high value of the serum SP-D prior to SBRT treatment develop severe RP (grade 4 or 5) at a relatively high rate. There was no correlation between dose-volume histogram parameters and severe RP events $(24,25)$. The present study identified that an increase in the serum SP-D level was associated with the occurrence of RP. However, grade 2-3 RP was also significantly associated with dose-volume histogram parameters, including mean lung dose and V20, in the present study. This data suggests that grade 2-3 RP are associated with dose-volume histogram parameters, whereas fatal RP (grade 5) is not. This may vary depending on other patient characteristics, including the interstitial lung condition reflected by the increased SP-D level. RP grade $>3$ was not encountered in the present study; however, it may be important to monitor SP-D to prevent severe adverse reactions.

Sasaki et al (7) hypothesized that serum SP-D monitoring is a practical and useful method for the early detection of RP. However, its use is limited to patients with normal levels of serum SP-D prior to the initiation of RT, because it was not predictive for patients with values outside of the normal range (7). We concur that measuring the eNO levels may also be valuable, particularly for high-risk patients with high levels of SP-D and/or an interstitial pneumonitis shadow (15). For example, in our previous study, lethal RP occurred in a patient with squamous cell lung cancer (T2N1M0) and idiopathic interstitial pneumonitis (15). The patient exhibited an elevation in eNO levels to 30-50 Gy, followed by a decrease to 10-20 Gy, twice the minimum value. The patient also exhibited an abnormal shadow on CT examination at $50 \mathrm{~Gy}$. RT treatment was discontinued; however, the patient developed fatal RP (15). Sasaki et al (7) have also identified a notable pattern of SP-D variation. In 3 patients with primary lung adenocarcinoma that developed RP, the initial serum SP-D levels were over twice the upper normal limit, but decreased following RT. A similar phenomena was observed in the present study in 2 patients with high serum SPD level prior to RT. The level was reduced by RT and then resurged to abnormally high values after a month; the RP was then observed after three months (Fig. 3). A potential hypothesis from this observation is that the hyperproduction of SP-D, the interstitial lung high-risk factor for RP, may be suppressed by RT and become resurgent following the onset of RP. 
As eNO is a non-specific inflammatory marker, numerous other factors, including corticosteroid use, smoking and diet, may have effects on eNO, causing difficulty in the interpretation of eNO variations. The response of the lung to radiation may also vary between patients. Zhang et al (27) demonstrated that genetic variants of NOS 2 may serve as reliable predictors for RP. The study genotyped a cohort of 301 patients for 21 single nucleotide polymorphisms (SNPs) in the NOS2 gene. The multivariate analysis identified that 3 SNPs (rs2297518, rs1137933 and rs16949) in NOS2 were significantly associated with the risk of RP. Thus, a multiple biomarker analysis (including SP-D, Krebs von den Lungen-6, transforming growth factor- $\beta 1$, IL-6 and eNO) including genotype analysis could be a successful approach for further study.

There are several limitations to the present study. Firstly, it was a small study concerning a small number of patients with a limited follow-up duration. Secondly, as no single biomarker was adequate for the prediction of RP, only the best timing for assessment and the best combination of biomarkers could be explored. Finally, a good method for identifying patients with a high risk of RP is required for serial biomarker monitoring.

In conclusion, obtaining the eNO ratio is a useful RP monitoring tool, but does not predict the occurrence of RP in the present setting. Serum SP-D level may be a potential predictor for the detection of RP risk.

\section{Acknowledgements}

The present study was supported in part by a Research Grant for Science and Cancer from the Ministry of Education, Culture, Sports, Science and Technology, Japan (Kiban C grant no. JP24591848).

\section{References}

1. Hart CM: Nitric oxide in adult lung disease. Chest 115: 1407-1417, 1999.

2. Alving K, Weitzberg E and Lundberg JM: Increased amount of nitric oxide in exhaled air of asthmatics. Eur Respir J 6 : $1368-1370,1993$.

3. Dweik RA, Boggs PB, Erzurum SC, Irvin CG, Leigh MW, Lundberg JO, Olin AC, Plummer AL and Taylor DR; American Thoracic Society Committee on Interpretation of Exhaled Nitric Oxide Levels (FENO) for Clinical Applications: An official ATS clinical practice guideline: Interpretation of exhaled nitric oxide levels (FENO) for clinical applications. Am J Respir Crit Care Med 184: 602-615, 2011.

4. Dummer JF, Epton MJ, Cowan JO, Cook JM, Condliffe R, Landhuis CE, Smith AD and Taylor DR: Predicting corticosteroid response in chronic obstructive pulmonary disease using exhaled nitric oxide. Am J Respir Crit Care Med 18: 846-852, 2009.

5. Tiev KP, Hua-Huy T, Kettaneh A, Allanore Y, Le-Dong NN, Duong-Quy S, Cabane J and Dinh-Xuan AT: Alveolar concentration of nitric oxide predicts pulmonary function deterioration in scleroderma. Thorax 67: 157-163, 2012.

6. Koizumi M, Yamazaki H, Toyokawa K, Yoshioka Y, Suzuki G, Ito M, Shinkawa K, Nishino K, Watanabe Y, Inoue T, et al: Influence of thoracic radiotherapy on exhaled nitric oxide levels in patients with lung cancer. Jpn J Clin Oncol 31: 142-146, 2001.

7. Sasaki R, Soejima T, Matsumoto A, Maruta T, Yamada K, Ota Y, Kawabe T, Nishimura H, Sakai E, Ejima Y and Sugimura K: Clinical significance of serum pulmonary surfactant proteins A and D for the early detection of RP. Int J Radiat Oncol Biol Phys 50: 301-307, 2001.

8. Yamashita H, Kobayashi-Shibata S, Terahara A, Okuma K, Haga A, Wakui R, Ohtomo K and Nakagawa K: Prescreening based on the presence of CT-scan abnormalities and biomarkers (KL-6 and SP-D) may reduce severe radiation pneumonitis after stereotactic radiotherapy. Radiat Oncol 5: 32, 2010.
9. International Union Against Cancer: TNM Classification of Malignant Tumours. Sobin LH, Gospodarowicz MK and Wittekind CH (eds). 7th edition. Wiley-Blackwell, Hoboken, NJ, 2009.

10. Cancer Therapy Evaluation Program: Common Toxicity Criteria. Version 2.0. http://ctep.cancer.gov/protocolDevelopment/electronic_applications/docs/ctcv20_4-30-992.pdf. Accessed April 30, 1999 .

11. Oxford University Press: Prescribing, recording, and reporting photon-beam intensity-modulated radiation therapy (IMRT). J ICRU 10: Report 83, 2010.

12. Cancer Therapy Evaluation Program: Common Terminology Criteria for Adverse Events. CTCAEv 4.0. https://ctep.cancer.gov/ protocoldevelopment/electronic_applications/ctc.htm. Accessed September 14, 2016.

13. Guida G, Culla B, Scirelli T, Bellone G, Sciascia S, Brussino L, Novero D, Palestro G, Heffler E, Gavarotti P, et al: Exhaled nitric oxide and nitric oxide synthase expression in Hodgkin's disease. Int J Immunopathol Pharmacol 22: 1027-1034, 2009.

14. Holmkvist T, Erlanson M, Meriläinen P and Högman M: Exhaled nitric oxide is highly increased in a case of Hodgkin's disease. Acta Oncol (Madr) 42: 788-789, 2003.

15. Wewel AR, Crusius JA, Gatzemeier U, Heckmayr M, Becher G, Magnussen H, Jörres RA and Holz O: Time course of exhaled hydrogen peroxide and nitric oxide during chemotherapy. Eur Respir J 27: 1033-1039, 2006

16. Liu CY, Wang CH, Chen TC, Lin HC, Yu CT and Kuo HP: Increased level of exhaled nitric oxide and up-regulation of inducible nitric oxide synthase in patients with primary lung cancer. Br J Cancer 78: 534-541, 1998.

17. Enache I, Noel G, Jeung MY, Meyer N, Oswald-Mammosser M, Urban-Kraemer E, Schumacher C, Geny B, Quoix E and Charloux A: Can exhaled NO fraction predict radiotherapy-induced lung toxicity in lung cancer patients? Radiat Oncol 7: 117, 2012.

18. Toyokawa K, Yamazaki H, Koizuni M, Inoue $\mathrm{T}$ and Inoue $\mathrm{T}$ : Assessment of exhaled NO concentration in monitoring RP in patient who underwent thoracic radiotherapy for lung cancer. Nihon Igaku Hoshasen Gakkai Zasshi 61: 347-349, 2001 (In Japanese)

19. Koizumi M, Yamazaki H, Toyokawa K, Ozeki S, Matsumura S, Inoue $\mathrm{T}$ and Inoue $\mathrm{T}$ : Influence of in vitro radiation on changes in nitric oxide in rat macrophages and smooth muscle cells. Anticancer Res 23: 331-334, 2003.

20. McCurdy MR, Wazni MW, Martinez J, McAleer MF and Guerrero T: Exhaled nitric oxide predicts RP in esophageal and lung cancer patients receiving thoracic radiation. Radiother Oncol 101: 443-448, 2011.

21. Guerrero T, Martinez J, McCurdy MR, Wolski M and McAleer MF: Elevation in exhaled nitric oxide predicts for radiation pneumonitis. Int J Radiat Oncol Biol Phys 82: 981-988, 2012.

22. Moré JM, Eclov NC, Chung MP, Wynne JF, Shorter JH, Nelson DD Jr, Hanlon AL, Burmeister R, Banos P, Maxim PG, et al: Feasibility and potential utility of multicomponent exhaled breath analysis for predicting development of radiation pneumonitis after stereotactic ablative radiotherapy. J Thorac Oncol 9: 957-964, 2014.

23. Yamazaki H, Tang JT, Inoue T, Teshima T, Ohtani M, Ikeda H, Itou M, Takeuchi $\mathrm{E}$ and Inoue T: Radiographic changes following radiotherapy in the patients with lung cancer. Is the irradiated area of the mediastinum in the simulation film a significant factor? Strahlenther Onkol 171; 272-277, 1995.

24. Yamashita H, Takahashi W, Haga A and Nakagawa K: Radiation pneumonitis after stereotactic radiation therapy for lung cancer. World J Radiol 6: 708-715, 2014.

25. Matsuno Y, Satoh H, Ishikawa H, Kodama T, Ohtsuka M and Sekizawa K: Simultaneous measurements of KL-6 and SP-D in patients undergoing thoracic radiotherapy. Med Oncol 23: 75-82, 2006.

26. Takahashi H, Imai Y, Fujishima T, Shiratori M, Murakami S, Chiba $\mathrm{H}$, Kon H, Kuroki Y and Abe S: Diagnostic significance of surfactant proteins A and D in sera from patients with radiation pneumonitis. Eur Respir J 17: 481-487, 2001.

27. Zhang J, Li B, Ding X, Sun M, Li H, Yang M, Zhou C, Yu H, Liu $\mathrm{H}$ and $\mathrm{Yu}$ G: Genetic variants in inducible nitric oxide synthase gene are associated with the risk of radiation-induced lung injury in lung cancer patients receiving definitive thoracic radiation. Radiother Oncol 111: 194-198, 2014. 